\title{
Report of Special Commission 3 of IAG
}

\author{
Erwin Groten, chairman \\ Institute of Physical Geodesy, Technical University of Darmstadt, \\ Peterestr. 13, 64287, Darmstadt, Germany
}

\begin{abstract}
Since the last presentation of SC-3 on numerical values of fundamental geodetic parameters at the IAU General Assembly at Kyoto in 1997 there were some conceptual as well as fundamental numerical changes. The four basic parameters of geodetic (ellipsoidal) reference systems (GRS) can no longer be considered as constant with time: $J_{2}$, $a, \omega$, and GM have to be replaced by clearly $\left( \pm 10^{-8}\right.$ or better) specified mean values or have to be associated with a specific epoch or, in case of GM, with specific reference frames ( $a=$ semi-major axis of Earth ellipsoid, $J_{2}=$ second degree zonal harmonic of geopotential, $\omega=$ spin of Earth rotation). In case of $\left(a, J_{2} \ldots\right)$ associated tidal reductions must be specifically defined in view of particular applications and significant differences between different tidal reduction types. Or we may replace " $a$ " by a quantity which is independent of tides like the geopotential at the geoid, $W_{0}$, where, however, also temporal changes are now discussed. The official geodetic reference systems such as GRS 80 and WGS 84 (revised in 97-form) are also no longer truly representing reality; a new system GRS 2000 is desired. We are, meanwhile, able to define and determine tidal and non-tidal (secular, periodic, aperiodic) variations of some fundamental geodetic parameters. Others are under investigation. New precession and/or nutation formulas to be adopted by IAU in 2000 or later would imply, again, changes in geodetic parameters such as $H=$ hydrostatic flattening. Those and related other consequences are considered.
\end{abstract}

\section{Introduction}

In comparison to the report of SC 3 presented at the IUGG-General Assembly at Birmingham (July 1999) which contains an overview on all relevant fundamental "constants" of geodesy there is relatively little change of numerical "current best estimates" so that the list of updates is relatively short.

On the other hand, there are some conceptual changes and revisions of accuracies of such "best estimates" which have to be taken into account. However, in agreement jointly with IAU those official parameters, which are no longer upto-date and which in the past were sometimes considered as constants, as well as their temporal changes have to be considered and revised.

Moreover, remeasurements of the "Newtonian Gravitational Constant" $G$ led to similar conclusions which were encountered a few years ago with the spin 
rate of the Earth's rotation $\omega$. In case of $\omega$ we had to truncate the official value because it did not reflect the temporal changes sufficiently.

In case of $G$ we are now more sceptical than before of the accuracy of previous determinations of "big $G$ " due to new determinations which deviate substantially from the previous determinations. However, more interesting to astronomy and to the IAU is the "geocentric" gravitational constant $G M$ where $M$ is the mass of the Earth. $G M$ is determined from satellite orbits using Kepler's third law and is one of the best determined constants (or parameters, if we include possible temporal changes).

Quantum physics is now of stronger impact to geodesy than in the past. For high precision time measurements, the "cold fountain" principle is now leading to better measurements of intervals than hydrogen masers. Moreover, results of macroscopic absolute gravimetry, which now reach accuracies of several microgals, have meanwhile been reconfirmed by "dropping atoms" methods where atomic interferomety leads to similar accuracies under laboratory conditions. Consequently, the direct measurement of "small $g$ " i.e., gravity at the surface of the Earth as vertical derivative of the geopotential including its variations with time and in space, will in the future be more reliably determined. Thus, for a conservative field, $-\vec{g}=\nabla W$, where $W=$ geopotential. Precise time-keeping is affected by this fact because the reduction of atomic clock measurements to the geoid in determing TAI involves the potential difference between the level of the observation site and the geoid. The exact determination of the geoid height of the clock location is no longer a problem.

It is important to realize that the potential $W_{0}$ at the geoid is independent of the tidal reductions whereas the semi-major axis of the Earth's ellipsoid (in the best fitting sense) depends significantly on the underlying tidal regime. As different tidal systems are necessary for different applications and purposes (which is not always well-understood in the scientific community) we are confronted with various (basically three) numerical values of the semi-major axis of the Earth ellipsoid which may lead to confusion. We may distinguish "mean tide", "tide free" and "zero frequency tide" values.

\section{A new Geodetic Reference System}

Meanwhile global satellite altimetry in combination with continental height systems allows us to determine $W_{0}$ with accuracy comparable to the accuracy of the semi-major axis $a$. In 1980 the consistent ellipsoidal (in the sense of PizzettiSomigliana) Geodetic Reference System was derived from the four parameters

$\left(a, G M, J_{2}, \omega\right)$

where $J_{2}$ takes the role of the flattening $f$. It represents the second zonal harmonic of the geopotential and is basically a function of $f$; moreover, it is directly derived from satellite orbit analysis. Theoretically, these four parameters are independent of each other. They consistently and uniquely determine the GRS 80 including its external spheroidal gravity field. 

value

Bursa et al. (1999) have meanwhile determined a new rounded numerical

$W_{0}=(62636856.0 \pm 0.5) m^{2} s^{-2}$

for the time 1993-1999. No significant secular trend was detected for the mean sea surface (a major part of the Earth's surface) during that time. All major continental height systems were included in the evaluation besides the sea surface using Topex/Poseidon data. This value is indeed independent of the underlying tidal regime whereas, depending on the specific tidal correction, the semi-major. axis varies by $20 \mathrm{~cm}$. Depending on the "inverted barometer" correction to be applied to the sea surface, $W_{0}$ varies by $0.33 \mathrm{~m}^{2} \mathrm{~s}^{-2}$. The authors, however, finally preferred to omit the "inverted barometer" hypothesis.

Grafarend and Ardalan (1999) computed a new Geodetic Reference Datum GRD 2000 in the Somigliana-Pizzetti-sense as described above. However, they adopted

$W_{0}=(62636855.72 \pm 0.5) m^{2} s^{-2}$

based on the Finnish height datum which differs by only $1.8 \mathrm{~cm}$ from the global geoid (Bursa et al., 1999). If we assume that, due to systematic perturbations, any (absolute) accuracy of better than $5 \mathrm{~cm}$ is purely formal (Ries, priv. comm., 2000 ) in satellite altimetry of the Topex-Poseidon type, a deviation of $1.8 \mathrm{~cm}$ can be ignored.

It appears, consequently, optimal to use the following parameters for a new GRS 2000:

$W_{0}=(62636856.0 \pm 0.5) \mathrm{m}^{2} \mathrm{~s}^{-2}$ (rounded)

$G M=(398600441.8 \pm 0.8) \mathrm{m}^{3} \mathrm{~s}^{-2}$ (Bursa et al., 1999)

$\omega=7292115 \times 10^{-11} \mathrm{rad} \mathrm{s}^{-1}$ (nominal)

$J_{2}=\left(-4.84165371736 \times 10^{-4}\right) \pm\left(3.56 \times 10^{-11}\right)$ ("tide-free"), EGM 96

The IAG Executive Committee at Birmingham in 1999 considered it "premature" to introduce GRS 2000 now but in view of the new system to be adopted by IAU in 2000 or 2001 a new GRS 2000 , perhaps based on updated numerical values, may be appropriate. The semi-major and semi-minor axes of such a GRS 2000 would deviate from those of GRS 80 by about $0.4 \mathrm{~m}$.

The interrelation between these four parameters is relatively weak; $W_{0}$ depends slightly on $G M$. Other parameters such as the hydrostatic flattening of the Earth can only be well-determined and updated when new nutation-precession parameters have been adopted by the IAU.

\section{Conclusions}

For astronomical purposes ellipsoidal models appear sufficient so the discussion of higher-degree approximations, as presently considered in geodesy, is not necessary here.

A fundamental problem lies in the fact that $G M, a$ and $W_{0}$ are more or less interrelated (pseudo-observations) and not, as assumed in theory of the 
Somigliana-Pizzetti concept, strictly independent. When $W_{0}$ was introduced as a primary constant, an improved determination (by satellite altimetry, tide gauge networks and repeat GPS) would be needed in order to verify the present (1999) best estimate of $W_{0}=(162636856.3 \pm 0.5) \mathrm{m}^{2} \mathrm{~s}^{-2}$ primarily based on the determination of J. Ries. The zero-tide and tide-free solutions appear to be the most appropriate for astrometry, in general.

Consequently, our latest list of fundamental parameters, reconciled with IERS Conventions in 1999, now needs only little and minor revisions of the numerical values, but rather numerous specifications.

The official introduction of a Geodetic Reference System "GRS 2000" is strongly recommended in view of the significant deviations of up-to-date ellipsoidal gravity fields of the Earth from GRS 80 as an official reference system. An obstacle to GRS 2000 is the fact that only recently WGS 84 was officially adopted by international navigation authorities (air and sea navigation) where WGS 84 is almost identical with GRS 80 . The updated list of "constants" presented at Birmingham in 1999 is given in appendices A and B.

\section{References}

Bursa M., Kouba, J., Kumar, M., Müller, A., Radej, K., True, S.A., Vatrt, V., Vojtiskova, M., 1999, Geoidal Geopotential and World Height System, Studia Geophysica, 43, 327-337.

Grafarend, E.W., Ardalan, A.A., 1999, World Geodetic Datum 2000, J. of Geodesy, 73, 611-623.

Appendix A

\section{A1 Current (2000) best estimates of the parameters of common rel- evance to astronomy, geodesy, and geodynamics}

SI units are used throughout (with the exception of the TDB-value below (4)). (SI-values can be associated with TCB or TCG).

- velocity of light in vacuum

$$
c=299792458 \mathrm{~ms}^{-1}
$$

- Newtonian gravitational constant

$$
G=(6672.59 \pm 0.30) \times 10^{-14} \mathrm{~m}^{3} \mathrm{~s}^{-2} \mathrm{~kg}^{-1} .
$$

- Geocentric gravitational constant (including the mass of the Earth's atmosphere); reconfirmed by J. Ries (1998, priv. comm.)

$$
G M=(398600441.8 \pm 0.8) \times 10^{6} \mathrm{~m}^{3} \mathrm{~s}^{-2} \text {. }
$$


For the new EGM 96 global gravity model $G M=398600441.5 \times 10^{6} \mathrm{~m}^{3} \mathrm{~s}^{-2}$ was adopted.

In TT units (Terrestrial Time) the value is

$G M=(398600441.5 \pm 0.8) \times 10^{6} \mathrm{~m}^{3} \mathrm{~s}^{-2}$.

Note that if expressed in old TDB units (solar system Barycentric Dynamical Time), the value is

$G M=398600435.6 \times 10^{6} \mathrm{~m}^{3} \mathrm{~s}^{-2}$.

Based on well-known transformation formulas we may relate GM in SI-units to TT/TCG/TCB; see IERS Convention 1996 p. 85. The well-known secular term was not originally included in the $\mathrm{GM}(\mathrm{E})$-analysis. Therefore it was related to TT, not to SI or (TCG, TCB). As satellite analyses still occur without the secular term, GM(E) in TT is still of geodetic interest. GM(E) = GM of the Earth.

- Mean angular velocity of the Earth's rotation

$\omega=7292115 \times 10^{-11} \mathrm{rads}^{-1}$.

Table 1. Mean angular velocity of the Earth's rotation 1978-1999.

\begin{tabular}{|c|c|c|c|c|}
\hline Year & $\omega\left[10^{-11} \mathrm{rad} \mathrm{s}^{-1}\right]$ & Year & $\omega\left[10^{-11} \mathrm{rad} \mathrm{s}^{-1}\right]$ & Mean LOD [ms/day $]$ \\
\hline $\min : 1978$ & 7292114.903 & 1995 & 7292114.952 & - \\
\hline max: 1999 & 292115.063 & 1996 & 7292114.992 & - \\
\hline & & 1997 & 7292114.991 & - \\
\hline & & 1998 & 7292115.031 & 1.37 \\
\hline & & 1999 & 7292115.063 & 0.99 \\
\hline
\end{tabular}

- Long-term variation in $\omega$

$d \omega / d t=(-4.5 \pm 0.1) \times 10^{-22} \mathrm{rad} \mathrm{s}^{-2}$.

This observed average value is based on two actual components:

a) due to tidal dissipation

$(d \omega / d t)_{t i d a l}=(-6.1 \pm 0.4) \times 10^{-22} \mathrm{rad} \mathrm{s}^{-2}$.

This value is commensurate with a tidal deceleration in the mean motion of the Moon $n$

$d n / d t=(-25.88 \pm 0.5) \operatorname{arcsec} c y^{-2}$.

b) non-tidal in origin 
$(d \omega / d t)_{\text {non-tidal }}=(+1.6 \pm 0.4) \times 10^{-22} \mathrm{rad} \mathrm{s}^{-2}$.

- Second-degree zonal geopotential (Stokes) parameter (tide-free, fully normalized, Love number $k_{2}=0.3$ adopted), in agreement with EGM 96,

$\bar{J}_{2}=4.84165371736 \times 10^{-4} \pm 3.56 \times 10^{-11}$.

To be consistent with the I.A.G. General Assembly Resolution 16, 1983 (Hamburg), the indirect tidal effect on $J_{2}$ should be included. Then in the zero-frequency tide system (JGM-3)

$$
J_{2}=(1082635.9 \pm 0.1) \times 10^{-9} .
$$

Table 2. The Stokes second-degree zonal parameter; marked with a bar: fully normalized; $k_{2}=0.3$ adopted for the tide-free system.

\begin{tabular}{|c|ll|lr|}
\hline Geopotential model & \multicolumn{2}{|c|}{ Zero-frequency tide system } & \multicolumn{2}{|c|}{ Tide-free } \\
& $\bar{J}_{2}\left[10^{-6}\right]$ & $J_{2}\left[10^{-6}\right]$ & $\bar{J}_{2}\left[10^{-6}\right]$ & $J_{2}\left[10^{-6}\right]$ \\
\hline JGM-3 & 484.16951 & 1082.6359 & 484.16537 & 1082.6267 \\
\hline EGM 96 & & & 484.16537 & \\
& & & & \\
\hline
\end{tabular}

- Long-term variation in $J_{2}$

$d J_{2} / d t=-(2.6 \pm 0.3) \times 10^{-9} c y^{-1}$.

- second-degree sectorial geopotential (Stokes) parameters (conventional, not normalized, geopotential model JGM-3)

$$
\begin{aligned}
& J_{2}^{2}=(1574.5 \pm 0.7) \times 10^{-9}, \\
& S_{2}^{2}=-(903.9 \pm 0.7) \times 10^{-9}, \\
& J_{2,2}=\sqrt{\left[\left(J_{2}^{2}\right)^{2}+\left(S_{2}^{2}\right)^{2}\right]}=(1815.5 \pm 0.9) \times 10^{-9} .
\end{aligned}
$$

Table 3. The Stokes second-degree sectorial parameters. marked with a bar: fully normalized

\begin{tabular}{|c|c|c|}
\hline Geopotential model & $\bar{C}_{2}^{2}\left[10^{-6}\right]$ & $\bar{S}_{2}^{2}\left[10^{-6}\right]$ \\
\hline JGM-3 & 2.43926 & -1.40027 \\
EGM 96 & 2.43914 & -1.40017 \\
\hline
\end{tabular}


Only the last decimal is affected by the standard deviation.

For EGM 96 Marchenko and Abrikosov (1999) found more detailed values:

Table 4. Parameters of the linear model of the potential of 2nd degree.

\begin{tabular}{|l|l|l|}
\hline Harmonic coefficient & Value of coefficien $\times 10^{-6}$ & Temporal variation $\times 10^{11}\left[\mathrm{yr}^{-1}\right]$ \\
\hline $\bar{C}_{20}=-\bar{J}_{2}$ & -484.165371736 & 1.16275534 \\
$\bar{C}_{21}$ & -0.00018698764 & -0.32 \\
$\bar{S}_{21}$ & 0.00119528012 & 1.62 \\
$\bar{C}_{22}=-\bar{J}_{2}^{2}$ & 2.43914352398 & -0.494731439 \\
$\bar{S}_{22}$ & -1.40016683654 & -0.203385232 \\
\hline
\end{tabular}

- Coefficient $H$ associated with the precession constant

$H=(C-0.5(A+B)) / C=(3273763 \pm 20) \times 10^{-9}$.

- The geoidal potential $W_{0}$ and the geopotential scale factor $R_{0}=G M / W_{0}$ recently derived by Bursa et al. (1998) read

$W_{0}=(62636855.611 \pm 0.5) \mathrm{m}^{2} \mathrm{~s}^{-2}$,

$R_{0}=(6363672.58 \pm 0.05) m$,

$W_{0}=(62636856.4 \pm 0.5) m^{2} s^{-2}$ J. Ries (priv. comm, 1998) found globally.

If $W_{0}$ is preserved as a primary constant the discussion of the ellipsoidal parameters could become obsolete as the Earth ellipsoid is basically an artifact. Modelling of the altimeter bias and various other error influences affect the validity of $W_{0}$-determination. The variability of $W_{0}$ and $R_{0}$ was studied by Bursa et al. (1998) recently; they detected interannual variations of $W_{0}$ and $R_{0}$ amounting to $2 \mathrm{~cm}$.

The relativistic corrections to $W_{0}$ were discussed by Kopejkin (1991). See his formulas (67) and (77) where tidal corrections were included. Whereas he proposes average time values, Grafarend insists in corrections related to specific epochs in order to illustrate the time-dependence of such parameters as $W_{0}, G M, J_{n}$, which are usually, in view of present accuracies, still treated as constants in contemporary literature.

Based on recent GPS data, Grafarend and Ardalan (1997) found locally (in the Finnish Datum for Fennoscandia) $W_{0}=(6263685.58 \pm 0.36) \mathrm{kgal} \mathrm{m}$.

The temporal variations were discussed by Wang and Kakkuri (1998), in general terms.

- Mean equatorial gravity in the zero-frequency tide system 
$g_{e}=(978032.78 \pm 0.2) \times 10^{-5} \mathrm{~ms}^{-2}$.

- Equatorial radius of the Reference Ellipsoid (mean equatorial radius of the Earth) in the zero-frequency tide system (Bursa et al., 1998)

$a=(6378136.62 \pm 0.10) m$.

- The corresponding value in the mean tide system (the zero-frequency direct and indirect tidal distortion included) comes out as

$a=(6378136.72 \pm 0.10) m$,

and the tide-free value

$a=(6378136.59 \pm 0.10) m$.

The tide free-value adopted for the EGM-96 gravity model reads

$a=6378136.3 \mathrm{~m}$.

- Polar flattening computed in the zero-frequency tide system, (adopted $G M$, $\omega$, and $J_{2}$ in the zero-frequency tide system)

$1 / f=298.25642 \pm 0.00001$.

The corresponding value in the mean tide system comes out as

$1 / f=298.25231 \pm 0.00001$,

and the tide-free

$1 / f=298.25765 \pm 0.00001$.

- Equatorial flattening (geopotential model $J G M-3$ )

$1 / \alpha_{1}=91026 \pm 10$

- Longitude of major axis of the equatorial ellipse, geopotential model $J G M-3$

$\Lambda_{a}=\left(14.9291^{\circ} \pm 0.0010^{\circ}\right) W$.

In view of the small changes (see Table 3 ) of the second degree tesserals it is close to the value of EGM 96. We may raise the question whether we should keep the reference ellipsoid in terms of GRS 80 (or an alternative) fixed and focus on $W_{0}$ as a parameter to be essentially better determined by satellite altimetry, where however the underlying concept (inverted barometer, altimeter bias etc.) has to be clarified.

- Coefficient in potential of centrifugal force 
Table 5. Equatorial flattening $\alpha_{1}$ and $\Lambda_{a}$ of major axis of equatorial ellipse.

\begin{tabular}{|c|c|c|}
\hline Geopotential model & $1 / \alpha_{1}$ & $\Lambda_{a}[$ deg. $]$ \\
\hline$J G M-3$ & 91026 & $14.9291 W$ \\
\hline
\end{tabular}

$q=\left(\omega^{2} a^{3}\right) /(G M)=(3461391 \pm 2) \times 10^{-9}$.

Computed by using values (3), (5) and $a=6378136.6$.

- Principal moments of inertia (zero-frequency tide system), computed using values (11), (15), (3), (2) and (16)

$$
\begin{aligned}
& (C-A) /\left(M a_{0}^{2}\right)=J_{2}+2 J_{2,2}=(1086.267 \pm 0.001) \times 10^{-6}, \\
& (C-B) /\left(M a_{0}^{2}\right)=J_{2}-2 J_{2,2}=(1079.005 \pm 0.001) \times 10^{-6}, \\
& (B-A) /\left(M a_{0}^{2}\right)=4 J_{2,2}=(7.262 \pm 0.004) \times 10^{-6} \\
& M a_{0}^{2}=\left(G M a_{0}^{2}\right) / G=(2.43014 \pm 0.00005) \times 10^{38} \mathrm{~kg} \mathrm{~m}^{2}, \\
& \left(a_{0}=6378137 \mathrm{~m}\right) \\
& C-A=(2.6398 \pm 0.0001) \times 10^{35} \mathrm{~kg} \mathrm{~m}^{2} \\
& C-B=(2.6221 \pm 0.0001) \times 10^{35} \mathrm{~kg} \mathrm{~m}^{2}, \\
& B-A=(1.765 \pm 0.0001) \times 10^{33} \mathrm{~kg} \mathrm{~m}^{2} \\
& C /\left(M a_{0}^{2}\right)=J_{2} / H=(330701 \pm 2) \times 10^{-6} \\
& A /\left(M a_{0}^{2}\right)=(329615 \pm 2) \times 10^{-6} \\
& B /\left(M a_{0}^{2}\right)=(329622 \pm 2) \times 10^{-6} ; \\
& A=(8.0101 \pm 0.0002) \times 10^{37} \mathrm{~kg} \mathrm{~m}^{2}, \\
& B=(8.0103 \pm 0.0002) \times 10^{37} \mathrm{~kg} \mathrm{~m} 2 \\
& A=(8.0365 \pm 0.0002) \times 10^{37} \mathrm{~kg} \mathrm{~m}^{2}, \\
& \alpha=(C-B) / A=(327353 \pm 6) \times 10^{-8}, \\
& \gamma=(B-A) / C=(2196 \pm 6) \times 10^{-8}, \\
& \beta=(C-A) / B=(329549 \pm 6) \times 10^{-8}
\end{aligned}
$$




\section{A2 Primary geodetic Parameters, discussion}

It should be noted that parameters $a, f, J_{2}, g_{e}$, depend on the tidal system adopted. They have different values in tide-free, mean or zero-frequency tidal systems. However, $W_{0}$ and/or $R_{0}$ are independent of tidal system (Bursa, 1995). The following relations can be used:

$$
\begin{aligned}
& a(\text { mean })=a(\text { tide }- \text { free })+0.5\left(1+\kappa_{s}\right) R_{0}\left(\delta J_{2}\right) / \kappa_{s}, \\
& \alpha(\text { mean })=\alpha(\text { tide }- \text { free })+1.5\left(1+\kappa_{s}\right)\left(\delta J_{2}\right) / \kappa_{s} . \\
& a(\text { zero }- \text { frequency })=a(\text { tide }- \text { free })+0.5 R_{0} \delta J_{2}, \\
& \alpha(\text { zero }- \text { frequency })=\alpha(\text { tide }- \text { free })+1.5 \delta J_{2} .
\end{aligned}
$$$$
\kappa_{s}=0.9383 \text { is the secular Love number, }\left(\delta J_{2}\right. \text { is the zero-frequency tidal }
$$$$
\text { distortion in } \left.J_{2}\right) \text {. First, the internal consistency of parameters } a, W_{0},\left(R_{0}\right) \text { and }
$$
$g_{e}$ should be examined:

(i) If

$a=6378136.7 m$

is adopted as primary, the derived values are

$$
\begin{aligned}
& W_{0}=62636856.88 \mathrm{~m}^{2} \mathrm{~s}^{-2}, \\
& \left(R_{0}=6363672.46 \mathrm{~m}\right), \\
& g_{e}=978032.714 \times 10^{-5} \mathrm{~ms}^{-2}
\end{aligned}
$$

(ii) If

$$
\begin{aligned}
& W_{0}=(62636855.8 \pm 0.5) \mathrm{m}^{2} \mathrm{~s}^{-2}, \\
& R_{0}=(6363672.6 \pm 0.05) \mathrm{m},
\end{aligned}
$$

is adopted as primary, the derived values are (mean system)

$$
a=6378136.62 m,
$$

$g_{e}=978032.705 \times 10^{-5} \mathrm{~ms}^{-2}$.

(iii) If (18)

$$
g_{e}=(978032.78 \pm 0.2) \times 10^{-5} \mathrm{~ms}^{-2},
$$


is adopted as primary, the derived values are

$a=6378136.38 m$,

$W_{0}=62636858.8 \mathrm{~m}^{2} \mathrm{~s}^{-2}$,

$R_{0}=6363672.26 m$.

There are no significant discrepancies, the differences are about the standard errors.

However, the inaccuracy in (iii) is much higher than in (i) and/or (ii). That is why solution (iii) is irrelevant at present.

If the rounded value

$W_{0}=(62636856.0 \pm 0.5) m^{2} s^{-2}$,

$R_{0}=(6363672.6 \pm 0.1) m$

is adopted as primary, then the derived length of the semi-major axis in the mean tide system comes out as

$a=(6378136.7 \pm 0.1) m,($ for zero-tide: 6378136.6$)$,

which is just the rounded value (20), and (in the zero frequency tide system)

$g_{e}=(978032.7 \pm 0.1) \times 10^{-5} \mathrm{~ms}^{-2}$.

However, SC 3 recommends that, at present, GRS 1980 should be retained as the standard.

\section{A3 Consistent set of fundamental constants (1997)}

- Geocentric gravitational constant (including the mass of the Earth's atmosphere)

$G M=398600441.8 \pm 0.8) \times 10^{6} \mathrm{~m}^{3} \mathrm{~s}^{-2}$;

[value (3)]

- Mean angular velocity of the Earth's rotation

$\omega=7292115 \times 10^{-11} \mathrm{rad} \mathrm{s}^{-1}$

[value (5)] 
- Second-degree zonal geopotential (Stokes) parameter (in the zero-frequency tide system, Epoch 1994)

$J_{2}=(1082635.9 \pm 0.1) \times 10^{-9}$

[value (11)]

- Geoidal potential

$W_{0}=(62636856.0 \pm 0.5) m^{2} s^{-2}$,

[value (44)]

- Geopotential scale factor

$R_{0}=G M / W_{0}=(6363672.6 \pm 0.05) m$

[value (45)]

- Mean equatorial radius (mean tide system)

$a=(6378136.7 \pm 0.1) m$

[value (46)]

- Mean polar flattening (mean tide system)

$1 / f=298.25231 \pm 0.00001$

[value (23)]

- Mean equatorial gravity

$g_{e}=(978032.78 \pm 0.1) \times 10^{-5} \mathrm{~ms}^{-2}$,

[value (18)].

Grafarend and Ardalan (1999) have evaluated a (consistent) normal field based on a unique set of current best values of four parameters ( $W_{\circ}, \omega, J_{2}$ and $G M$ ) as a preliminary "follow-up" to the Geodetic Reference System GRS 80. It can lead to a level-ellipsoidal normal gravity field with a spheroidal external field in the Somigliana-Pizetti sense. By comparing the consequent values for the semi-major and semi-minor axes of the related equipotential ellipsoid with the corresponding GRS-80 axes (based on the same theory) the authors end up with axes which deviate by -40 and $-45 \mathrm{~cm}$, respectively from GRS 80 axes and within standard deviations from the current values such as in (21); but no $\mathrm{g}$-values are given until now.

\section{Appendix B}

B1 Zero-frequency tidal distortion in $J_{2}$

$\left(J_{2}=-C_{20}\right)$ 


$$
\begin{aligned}
& \delta J_{2}=\kappa_{s}\left[\left(G M_{L}\right) /(G M)\right]\left(\bar{R} / \Delta_{\oplus L}\right)^{3}\left(\bar{R} / a_{0}\right)^{2}\left(E_{2}+\delta_{2 L}\right)+ \\
& \kappa_{s}\left[\left(G M_{s}\right) /(G M)\right]\left(\bar{R} / \Delta_{\oplus S}\right)^{3}\left(\bar{R} / a_{0}\right)^{2}\left(E_{2}+\delta_{2 S}\right), \\
& E_{2}=-0.5+0.75 \sin ^{2} \epsilon_{0}, \\
& \delta_{2 L}=\frac{3}{4}\left(\sin ^{2} i_{L}-e_{L}^{2}\right)+\frac{9}{8} e_{L}^{2}\left(\sin ^{2} \epsilon_{0}-\sin ^{2} i_{L}\right), \\
& \delta_{2 S}=-\frac{3}{4} e_{S}^{2}\left(1-\frac{3}{2} \sin ^{2} \epsilon_{0}\right) \text {, } \\
& \bar{R}=R_{0}\left(\frac{25}{21} \nu^{3} q-\frac{10}{7} \nu^{2} J_{2}\right)^{1 / 5} \\
& G M_{L}=4902.799 \times 10^{9} \mathrm{~m}^{3} \mathrm{~s}^{-2} \text { (selenocentric grav. Const.), } \\
& G M_{S}=13271244.0 \times 10^{13} \mathrm{~m}^{3} \mathrm{~s}^{-2} \text {, } \\
& \Delta_{\oplus L}=384400 \mathrm{~km} \text { (mean geocentric distance to the Moon), } \\
& \Delta_{\oplus S}=1 A U=1.4959787 \times 10^{11} \mathrm{~m}, \\
& a_{0} \quad=6378137 \mathrm{~m} \text { (scaling parameter associated with } J_{2} \text { ), } \\
& \epsilon_{0} \quad=23^{\circ} 26^{\prime} 21^{\prime \prime} 4 \text { (obliquity of the ecliptic), } \\
& e_{L} \quad=0.05490 \text { (eccentricity of the orbit of the Moon), } \\
& i_{L} \quad=5^{\circ} 0^{\prime} 9 \text { (inclination of Moon's orbit to the ecliptic), } \\
& e_{S} \quad=0.01671 \text { (eccentricity of the heliocentric orbit of the Earth-Moon } \\
& \text { barycenter), } \\
& \nu \quad=a_{0} / R_{0}=1.0022729 \\
& \kappa_{s}=0.9383 \text { (secular-fluid Love number associated with the zero-frequency } \\
& \text { second zonal tidal term); } \\
& \delta J_{2}=-\delta C_{20}=\left(3.07531 \times 10^{-8}\right) \kappa_{s} \text { (conventional); } \\
& \delta \bar{J}_{2}=-\delta \bar{C}_{20}=\left(1.37532 \times 10^{-8}\right) \kappa_{s} \text { (fully normalized). } \\
& \mathrm{L}=\text { lunar } \\
& \mathrm{S}=\text { solar }
\end{aligned}
$$

\section{B2 Definition}

Because of tidal effects on various quantities, the tide-free, zero-frequency and mean values should be distinguished as follows:

- A tide-free value is the quantity from which all tidal effects have been removed.

- A zero-frequency value includes the indirect tidal distortion, but not the direct distortion.

- A mean tide value includes both direct and indirect permanent tidal distortions.

Acknowledgments. This report is basically an updated version of $\mathrm{M}$. Bursa's SC 3 report presented in 1995 with some new material added. 


\section{References}

Bursa, M., 1992, The four primary geodetic parameters, Studia geoph. et geod., 36, 199-206.

Bursa, M., 1995, Special Commission 3, Fundamental Constants (SCFC), Report submitted to IAG, IUGG Gen. Ass., Boulder, Travaux IAG, Tome 30, 370-384.

Bursa, M., Kouba, J., Radej, K., True, S.A., Vatrt, V., Vojtiskova, M., 1997, Monitoring geoidal potential on the basis of Topex/Poseidon altimeter data and EGM 96. Geodesy on the Move, IAG Sci. Ass., Rio de Janeiro, Sept. 3-9, Springer Verlag, 352-358.

Bursa, M., Radej, K., Sima, Z., True, S.A., Vatrt, V., 1997, Tests for accuracy of recent Geopotential models, Intern. Geoid Service, Bulletin 6, $167-188$.

Bursa, M., Kouba, J., Radej, K., True, S.A., Vatrt, V., Vojtiskova, M., 1998a, Monitoring Geoidal Potential on the Basis of Topex/Poseidon Altimeter Data, Aviso Altimetry Newsletters 6, 130-131.

Bursa, M., Kouba, J., Müller, A., Radej, K., True, S.A., Vatrt, V., Vojtiskova, M., 1998b, Determination of Geopotential Differences between local vertical Datums and realization of a world height system, Proc. Symp. IAG, München.

Bursa, M., Demianov, G.V., Yurkina, M.I., 1998c, On the determination of the earth's model - the mean equipotential surface, Studia geoph. et geod., 42, 467-471.

Bursa, M., Kouba, J., Radej, K., True, S.A., Vatrt, V., Vojtiskova, M., 1998d, Mean earth's equipotential surface from Topex/Poseidon altimetry, Studia geoph. et geod., 42, 459-466.

Bursa, M., Kouba, J., Radej, K., True, S.A., Vatrt, V., Vojtiskova, M., 1998e, Final Report on testing accuracy of geopotential models EGMX05, EGM 96. Intern. Geoid Service, Bulletin N. 7.

Bursa, M., Kouba, J., Kumar, M., Müller, A., Radej, K., True, S.A., Vatrt, V., Vojtiskova, M., 1999, Geoidal geopotential and world height system, Studia geoph. et geod., 43, 327-337.

Cheng, M.K., Eanes, R.J., Tapley, B.D., 1992, Tidal deceleration of the Moon's mean motion, Geophys. J. Int., 108, 401-409.

Defraigne, P., Dehant, V., 1996, Toward new non-rigid Earth nutations, Proc. Journ. de Référence 1995, ed. N. Capitaine, Warsaw, 45-52.

Dickey, J.O., Bender, P.L., Faller, J.E., Newhall, XX, Ricklefs, R.L., Ries, J.G., Shelus, P.J., Veillet, C., Whipple, A.L., Wiant, J.R., Williams, J.G., Yoder, C.F., 1994, Lunar laser ranging: a continual legacy of the Apollo program, Science, 265, 482-490.

Fukushima, T., 1993, IAU Standards - What's it?, Proc. IAG. Gen. Ass. Beijing.

Fukushima, T., 1994, Time ephemeris. Proc. of 26th Symp. on "Cel. Mechanics", Tokyo, Japan, Jan 12-13, eds. H. Kinoshita and H. Nakai, 149-159. 
Grafarend, E., Sanso, F., 1984, The multibody space-time geodetic boundary value problem and the Honkasalo term, Geophys. J. R. astr. Soc., 78, 255-275.

Grafarend, E., Ardalan, A.A., 1997, $W_{0}$ : an estimate in the Finnish Height Datum N60, epoch 1993.4, from twenty-five GPS points of the Baltic Sea Level Project. J. Geodesy, 71, 673-679.

Grafarend, E., Ardalan, A.A., 1999, The form parameters of the Somigliana-Pizetti level ellipsoid from current best estimates of fundamental geodetic parameters based on a functional analytical review of the Somigliana-Pizetti gravitational field, J. Geodesy, (in press).

Grafarend, E., Ardalan, A.A., 1999, World Geodetic Datum 2000, J. Geodesy, 73, 611-623.

Groten, E., 1994, A comment on fundamental geodetic constants. IAG-SCFC 1991-1995, pres. at IAU General Ass. The Hague.

Groten, E., 1994, Global tidal parameters, Studia geoph. et geod., 38, 221-234.

Kinoshita, H., 1994, Is the equatorial radius of the Earth a primary constant, or a defining constant?, Studia geoph. et geod., 38, 109-116.

Kopejkin, S.M., 1991, Relativistic manifestations of gravitational fields in gravimetry and Geodesy, Manuscripta Geodaetica, 16, 301-312.

Lemoine et al., 1996, The development of the NASA GSFC and NIMA joint geopotential model, Paper presented at IAG Symposium, Tokyo, Sept. 1996.

Marchenko, A.N. and Abrikosov, O.A., 1999, Evolution of the Earth principal axes and moments of inertia, J. Geodesy, (in press).

McCarthy, D.D., ed., 1992, IERS Standards. Technical Note 13, Observatoire de Paris.

McCarthy D.D., 1996, IERS Conventions.

Nerem, R.S., Chao, B.F., Au, A.Y., Chan, J.C., Klosko, S.M., Pavlis, N.K., Williamson, R.G., 1993, Temporal variations of the Earth's gravitational field from satellite laser ranging to LAGEOS, Geophys. Res. Let., 20, 595-598.

Nerem, R.S., Lerch, F.J., Williamson, R.G., Klosko, S.M., Robbins, J.W., Patel, G.B., 1994, Gravity model improvement using the DORIS tracking system on the SPOT 2 satellite, J. Geophys. Res., 89, B2, 2791-2813.

Nerem, R.S., Lerch, F.J., Marshall, J.A., Pavlis, E.C., Putney, B.H., Tapley, B.D., Eanes, R.J., Ries, J.C., Schutz, B.E., Shum, C.K., Watkins, M.W., Klosko, S.M., Chan, J.C., Luthcke, S.B., Patel, G.B., Pavlis, N.K., Williamson, R.G., Rapp, R.H., Biancale, R., Nouel, F., 1994, Gravity model development for TOPEX/POSEIDON: Joint gravity models 1 and 2, J. Geophys. Res., 99, C12, 24, 421-24, 447.

Nesvorný, D., Śíma, Z., 1994, Refinement of the geopotential scale factor $R_{0}$ on the satellite altimetry basis, Earth, Moon, and Planets, 65, 79-88.

NIMA, National Imagery and Mapping Agency, 1997, Technical Report TR 8350.2, Third Edition, Department of Defense, World Geodetic System, 1984, Its Definition and Relationships with Local Geodetic Systems. 
Rapp, R.H., 1987, An estimate of equatorial gravity from terrestrial and satellite data, Geophys. Res. Lett., 14, 730-732.

Rapp, R.H., 1993, Geoid undulation accuracy. IEEE Transaction on geoscience and remote sensing, 31, 365-370.

Rapp, R.H., Yi, Y., Wang, Y.M., 1994, Mean sea surface and geoid gradient comparison with Topex altimeter data, J. Geophys. Res., 99, C12, 24, $657-24,667$.

Ries, J.C., Eanes, R.J., Shum, C.K., Watkins, M.M., 1992, Progress in the determination of the gravitational coefficient of the Earth, Geophys. Res. Lett., 19, 529-31.

Ries, J.C., 1998, Personal communications.

Stephenson, F.R., Morrison, L.V., 1994, Long-term fluctuations in the Earth's rotation: $700 \mathrm{BC}$ to AD 1990, Phil. Trans. Royal Soc. (London) Ser. $A$.

Wang, Z., Kakkuri, J., 1998, The time dependency of gravity potential on the geoid, J. Geodesy, (in press).

Williams, J.G., Newhall, XX, Dickey, J.O., 1993, In Contributions of space geodesy to geodynamics: Earth Dynamics, eds. D.E. Smith, D.L. Turcotte, Geodynamics series 24, AGU, Washington, 83.

Williams, J.G., 1994, In Contributions to the Earth's obliquity rate, precession and nutation, Astron. J., 108, 711-724.

Yan, H.J., Groten, E., 1994, The celestial and terrestrial departure points and their various aspects in geodesy and astrometry, Studia geoph. et geod., 38, 117-130.

Yurkina, M.I., 1993, Determination of Stokes' constants respecting zero-frequency tidal term due to the Moon and the Sun, Studia geoph. et geod., 37, 317-325. 